\title{
Análise da síndrome de "burnout" e das estratégias de "coping" em atletas brasileiros de vôlei de praia
}

CDD. 20.ed. 796.011

796.325

\author{
Lenamar Fiorese VIEIRA* \\ Nayara Malheiros CARRUZO* \\ Paulo Vitor Suto AIZAVA* \\ Patrícia Aparecida Gaion RIGONI* \\ *Universidade Estadual
de Maringá.
}

.

\section{Resumo}

Este estudo teve como objetivo analisar a síndrome de "burnout" e as estratégias de "coping" de jogadores de vôlei de praia. Participaram 93 atletas, das categorias sub-21 e "open" do circuito brasileiro de vôlei de praia. Como instrumentos foram utilizados uma ficha com dados de identificação, Questionário de "Burnout" para Atletas e Inventário Atlético das Estratégias de "Coping". Para análise dos dados utilizou-se teste de Kolmogorov-Smirnov, coeficiente Alpha de Cronbach, teste 'U' de Mann-Whitney, teste de Kruskal-Wallis e coeficiente de correlação de Spearman, adotando $p<0,05$. Verificou-se correlação significativa e negativa entre as dimensões do "burnout" e estratégias de "coping". Para as dimensões do "burnout", apenas "ranking" obteve diferença significativa, enquanto para as estratégias de "coping", gênero, categoria, "ranking" e tempo de prática apresentaram diferenças significativas. Concluiu-se que quanto maior a síndrome de "burnout", menor é a utilização de estratégias de "coping" em atletas de vôlei de praia.

Palavras-chave: Psicologia do esporte; Emoções; Estafa profissional; Estratégias de enfrentamento.

\section{Introdução}

A síndrome de "burnout" tem sido considerada um grave problema que afeta profissionais de diferentes áreas, com repercussões físicas, psicológicas e sociais que podem gerar no indivíduo uma incapacidade total para a atividade ${ }^{1-4}$. Em atletas, além do impacto no rendimento esportivo, esse esgotamento derivado de uma reação ao estresse crônico pode levar ao abandono do esporte ${ }^{5-6}$.

Ainda que atletas possam sofrer da síndrome de "burnout" 7-8 e que este tema seja bastante documentado entre profissóes como professores, médicos e enfermeiros ${ }^{2}$, existe uma lacuna na literatura, sobretudo em língua portuguesa, a respeito da prevalência no meio esportivo 5 .

Além disso, a forma como os atletas avaliam, respondem e se comportam perante essas situaçōes, ou seja, suas estratégias de "coping", bem como as diferenças entre os gêneros e níveis de desempenho ainda não estão totalmente esclarecidas, tendo em vista as variações metodológicas entre os estudos, como o de ANSHEL et al. ${ }^{10}$ que comparou as estratégias de "coping" em função da raça e do gênero em um único evento estressante de atletas adultos, o de Nicholls et al. ${ }^{11}$, que analisou as estratégias de "coping" de atletas adolescentes de acordo com idade biológica, idade cronológica e gênero e, o estudo de RoSADO et al..$^{12}$, que investigou o impacto do gênero e do tempo de prática na relação entre satisfação e esgotamento em atletas portugueses de diferentes modalidades esportivas.

A maioria dos estudos envolvendo atletas investigou o estresse, sobretudo em situaçôes competitivas ${ }^{13}$, com esportes coletivos tradicionais como basquetebol $^{14}$, handebol e voleibol ${ }^{15}$ ou analisando atletas de diferentes modalidades esportivas em um único estudo, sem diferenciação por tipo de esporte ${ }^{16}$.

Com relação ao vôlei de praia, a pesquisa de STEFANELlo ${ }^{17}$, com uma dupla olímpica brasileira apontou que as principais fontes de estresse dos jogadores são os fatores relacionados à facilidade ou dificuldade da partida e que as estratégias mais empregadas para superar o estresse são as técnicas cognitivas. Apesar da relevância, este estudo foi de 
caso e avaliou o estresse, o que indica que, até onde sabemos, não existem estudos em língua portuguesa que tenham investigado a relação entre síndrome de "burnout" e estratégias de "coping" em atletas de vôlei de praia de diferentes categorias e gênero.

Nesse sentido, o objetivo do presente estudo foi analisar a síndrome de "burnout" e as estratégias

\section{Método}

\section{Amostra}

Este estudo de caráter descritivo ${ }^{18}$, teve como população alvo, todos os 144 atletas do Circuito Banco do Brasil de vôlei de praia, das categorias sub-21 e "open", de ambos os gêneros. A abordagem junto aos atletas e a aplicação dos questionários foram feitas no congresso técnico de uma das etapas do Circuito Brasileiro de Vôlei de Praia. Todos os atletas foram convidados a participar do estudo, no entanto, 93 consentiram, sendo 45 da categoria sub-21 (24 do gênero masculino e 21 do gênero feminino) e 48 atletas da categoria "open" (24 do gênero masculino e 24 do gênero feminino). A divisão dos atletas por "ranking" foi feita da seguinte forma: agrupou-se as duplas que ficaram entre o $1^{\circ}$ e o $4^{\circ}$ lugar por serem as duplas semifinalistas; as duplas entre $5^{\circ}$ a $8^{\circ}$ lugar por serem aquelas que não passaram das quartas de final (e, por isso, apresentaram a mesma pontuação); as duplas entre $9^{\circ}$ e $12^{\circ}$ lugar, por terem perdido os dois jogos da chave e as duplas acima do $13^{\circ}$ lugar, que foram as piores colocadas no campeonato. Já a divisão dos atletas em duas categorias segue os critérios da Confederação Brasileira de Voleibol. Este estudo faz parte de um projeto institucional da universidade, aprovado pelo Comitê Permanente de Ética em Pesquisa com Seres Humanos sob o Parecer $n^{\circ} 175 / 2007$. Para participação, todos os atletas assinaram o termo de consentimento livre e esclarecido.

\section{Procedimentos}

Para caracterização geral dos participantes foi utilizada uma ficha contendo dados referentes a gênero, idade, tempo de prática, categoria e "ranking" atual. Para análise da síndrome de "burnout", utilizou-se o Questionário de "Burnout" para Atletas, de RADEKE e MITH $^{19}$, validado para a língua portuguesa por PIRES et al. ${ }^{5}$, composto por 15 itens que avaliam a frequência de sentimentos relativos ao "burnout". Os de "coping" utilizadas por atletas, de ambos os gêneros, das categorias sub-21 e open, participantes do circuito brasileiro de vôlei de praia, tendo como hipóteses que os atletas da categoria open apresentam maior prevalência de síndrome de "burnout", mas se utilizam de mais estratégias de "coping" para lidar com essas situaçōes.

itens são agrupados em três subescalas: exaustão física e emocional, reduzido senso de realização esportiva e desvalorização da modalidade esportiva. As respostas são dadas em uma escala do tipo Likert que varia de "quase nunca" (1) a "quase sempre" (5) e os resultados são obtidos a partir da média aritmética das respostas dadas aos cinco itens correspondentes a cada uma das subescalas, e a um valor de "burnout" total calculado pela média aritmética de todos os 15 itens do instrumento, de modo que, quanto menor o valor, menos frequentes são os sentimentos relativos ao "burnout". O Alpha de Cronbach do teste foi de $\alpha=0,79$, demonstrando uma boa confiabilidade dos dados.

Para análise das estratégias de "Coping", utilizouse o Inventário Atlético das Estratégias de "Coping", desenvolvido por SMITH et al. ${ }^{20}$ e validado para língua portuguesa por Serpa e Palmeira ${ }^{21}$. Composto por 28 itens, cujas respostas se dão em uma escala Likert de quatro pontos, variando de (0) "quase nunca" a (3) "quase sempre", este instrumento apresenta sete subscalas que avaliam as seguintes competências psicológicas: a) rendimento máximo sob pressão; b) ausência de preocupações; c) confronto com a adversidade; d) concentração; e) formulação de objetivos; f) confiança e motivação para a realização e g) treinabilidade ou disponibilidade para aprendizagem a partir do treino. Os resultados são obtidos a partir das médias das subescalas e a média de todos os valores do instrumento indica o Índice de Confronto no Esporte, o qual, quanto maior o valor obtido, maior a capacidade de confronto perante a situação de competição. O Alpha de Cronbach de $\alpha$ $=0,75$ demonstrou uma boa confiabilidade do teste.

\section{Análise dos resultados}

Foram adotados o coeficiente Alpha de Cronbach para avaliar a confiabilidade entre os itens do questionário e o teste de Kolmogorov-Smirnov para verificar a normalidade da distribuição dos dados; 
como os dados não apresentaram distribuição normal, utilizou-se da mediana e intervalo interquartil (diferença entre o terceiro e o primeiro quartil) para descrição dos resultados, teste 'U' de Mann-Whitney para comparação entre as categorias e entre os gêneros, teste de Kruskal-Wallis para comparação entre os "rankings" e entre o tempo de prática e coeficiente de correlação de Spearman para correlacionar as estratégias de "coping" e as dimensões de "burnout". A significância adotada foi de $\mathrm{p}<0,05$.

\section{Resultados}

Dos 93 atletas investigados, 51,7\% ( $\mathrm{n}=48)$ eram do gênero masculino e pertenciam à categoria "open", com mediana de idade de 20 anos $(\mathrm{IIQ}=12)$ e tempo de prática com mediana de seis anos $(\mathrm{IIQ}=6)$. ATABELA 1 apresenta a comparação das dimensões do "burnout" em função do gênero, categoria, "ranking” e tempo de prática.

TABELA 1 - Comparação das dimensões de "burnout" em relação ao gênero, categoria, "ranking” e tempo de prática de atletas brasileiros de vôlei de praia.

\begin{tabular}{|c|c|c|c|c|c|}
\hline \multirow{2}{*}{\multicolumn{2}{|c|}{ Variáveis }} & \multicolumn{3}{|c|}{ "Burnout" } & \multirow[b]{2}{*}{$\begin{array}{l}\text { Valor total de } \\
\text { "burnout" }\end{array}$} \\
\hline & & $\begin{array}{c}\text { Exaustão física } \\
\text { e emocional }\end{array}$ & $\begin{array}{l}\text { Reduzido senso de } \\
\text { realização esportiva }\end{array}$ & $\begin{array}{c}\text { Desvalorização } \\
\text { da modalidade } \\
\text { esportiva }\end{array}$ & \\
\hline \multirow[t]{3}{*}{ Gênero } & & Md (IIQ) & Md (IIQ) & Md (IIQ) & Md (IIQ) \\
\hline & Masculino & $1,57(0,85)$ & $2,50(0,34)$ & $1,50(1,00)$ & $2,00(0,54)$ \\
\hline & Feminino & $1,27(0,85)$ & $2,58(0,67)$ & $1,75(1,50)$ & $2,07(0,67)$ \\
\hline \multicolumn{6}{|c|}{ Categoria } \\
\hline & Sub-21 & $1,43(0,57)$ & $2,50(0,67)$ & $1,50(1,50)$ & $2,00(0,60)$ \\
\hline & Open & $1,78(1,14)$ & $2,50(0,50)$ & $1,50(1,50)$ & $2,07(0,60)$ \\
\hline \multicolumn{6}{|c|}{ "Ranking" } \\
\hline & $1^{o}-4^{o}$ & $1,71(0,85)$ & $2,41(0,74)$ & $1,50(1,00)^{\mathrm{a}}$ & $2,03(0,64)$ \\
\hline & $5^{\circ}-8^{o}$ & $1,43(0,50)$ & $2,50(0,17)^{\mathrm{d}}$ & $1,00(0,50)^{\mathrm{b}}$ & $1,87(0,37)^{\mathrm{c}, \mathrm{e}}$ \\
\hline & $9^{\circ}-12^{\circ}$ & $2,00(1,28)$ & $2,50(0,84)$ & $2,50(2,00)^{\mathrm{a}, \mathrm{b}, \mathrm{f}}$ & $2,16(1,04)^{\mathrm{c}}$ \\
\hline & $>13^{\circ}$ & $1,57(0,85)$ & $2,67(0,50)^{d}$ & $1,75(1,50)^{\mathrm{f}}$ & $2,10(0,67)^{\mathrm{e}}$ \\
\hline \multicolumn{6}{|c|}{ Tempo de prática (anos) } \\
\hline & $1-5$ & $1,57(0,71)$ & $2,50(0,67)$ & $1,50(1,50)$ & $2,07(0,51)$ \\
\hline & $6-14$ & $1,57(1,00)$ & $2,50(0,58)$ & $1,00(1,00)$ & $1,93(0,60)$ \\
\hline & $>15$ & $2,00(1,28)$ & $2,67(0,50)$ & $2,25(1,50)$ & $2,20(1,00)$ \\
\hline
\end{tabular}

adiferença significativa $(p=0,003)$; bdiferença significativa $(p<0,0001)$; 'diferença significativa $(p=0,011)$; diferença significativa $(p=0,002)$; ediferença significativa $(p=0,015)$; diferença significativa $(\mathrm{p}$ $=0,016)$.
Observa-se na TABELA 2 que o gênero masculino utiliza mais das estratégias de "coping" de rendimento máximo sob pressão, concentração, formulação de objetivos, confiança e motivação para a realização, treinabilidade e índice de confronto no esporte do que o feminino $(\mathrm{p}<0,05)$.

Nota-se na TABELA 3 que a categoria sub21 utiliza com mais frequência de estratégias de “coping” relacionadas à ausência de preocupaçóes ( $\mathrm{p}$ $=0,033)$ e treinabilidade $(\mathrm{p}<0,0001)$, enquanto a categoria "open" realiza mais estratégias relacionadas ao rendimento máximo sob pressão $(\mathrm{p}=0,045)$.

Verificou-se na TABELA 4 que os atletas que estavam ranqueados entre o $5^{\circ}$ e $8^{\circ}$ lugar apresentaram maior estratégia de "coping" para rendimento máximo sob pressão, em relação aos atletas do 90 lugar em diante. 
Vieira LF, et al.

TABELA 2 - Comparação das estratégias de “coping” em relação ao gênero de atletas brasileiros de vôlei de praia.

${ }^{*} p<0,05$

\begin{tabular}{lccc}
\hline \multirow{2}{*}{ “Coping” } & \multicolumn{2}{c}{ Gênero } & \multirow{2}{*}{$\mathrm{p}$} \\
\cline { 2 - 3 } & Masculino & Feminino & \\
\hline Rendimento máximo sob pressão & Md (IIQ) & $1,62(1,25)$ & $0,019^{*}$ \\
Ausência de preocupaçóes & $2,00(0,75)$ & $1,25(1,00)$ & 0,106 \\
Confronto com adversidade & $1,50(1,00)$ & $1,75(0,83)$ & 0,076 \\
Concentração & $2,00(1,00)$ & $1,75(0,75)$ & $0,031^{*}$ \\
Formulação de objetivos & $1,75(1,00)$ & $2,00(0,38)$ & $0,012^{*}$ \\
Confiança e motivação para a realização & $2,25(0,75)$ & $2,12(0,75)$ & $0,002^{*}$ \\
Treinabilidade & $2,50(0,75)$ & $1,50(0,50)$ & $0,006^{*}$ \\
Índice de confronto no esporte & $2,25(1,50)$ & $6,71(1,64)$ & $<0,0001^{*}$ \\
\hline
\end{tabular}

TABELA 3 - Comparação das estratégias de “coping” em relação à categoria de atletas brasileiros de vôlei de praia.

${ }^{*} p<0,05$

\begin{tabular}{lccc}
\hline \multirow{2}{*}{ “Coping” } & \multicolumn{2}{c}{ Categoria } & \multirow{2}{*}{$\mathrm{p}$} \\
\cline { 2 - 3 } & Sub-21 & “Open” & \\
\hline Rendimento máximo sob pressão & $1,75(1,00)$ & $2,00(0,88)$ & $0,045^{*}$ \\
Ausência de preocupaçóes & $1,50(1,00)$ & $1,25(0,83)$ & $0,033^{*}$ \\
Confronto com adversidade & $1,75(0,92)$ & $2,00(0,75)$ & 0,254 \\
Concentração & $1,75(1,00)$ & $1,75(0,75)$ & 0,936 \\
Formulaçáo de objetivos & $2,25(1,00)$ & $2,25(0,50)$ & 0,649 \\
Confiança e motivação para a realização & $2,25(1,00)$ & $2,37(0,75)$ & 0,565 \\
Treinabilidade & $2,00(1,25)$ & $1,50(0,75)$ & $<0,0001^{*}$ \\
Índice de confronto no esporte & $7,29(2,57)$ & $7,57(1,43)$ & 0,623 \\
\hline
\end{tabular}

TABELA 4 - Comparação das estratégias de “coping” em relação ao “ranking” de atletas brasileiros de vôlei de praia.

\begin{tabular}{|c|c|c|c|c|c|}
\hline \multirow{3}{*}{ “Coping” } & \multicolumn{4}{|c|}{ "Ranking" } & \multirow{3}{*}{$\mathrm{p}$} \\
\hline & $1^{\mathrm{o}-}-4^{\mathrm{o}}$ & $5^{\circ}-8^{o}$ & $9^{\circ}-12^{\circ}$ & $>13^{\circ}$ & \\
\hline & Md (IIQ) & Md (IIQ) & Md (IIQ) & Md (IIQ) & \\
\hline \multirow[t]{2}{*}{ Rendimento máximo sob pressão } & $1,87(0,63)$ & $2,50(0,75)^{a, b}$ & $1,62(1,25)^{\mathrm{a}}$ & $2,00(1,00)^{\mathrm{b}}$ & $0,01^{\mathrm{a}}$ \\
\hline & & & & & $0,031^{\mathrm{b}}$ \\
\hline Ausência de preocupaçóes & $1,12(1,12)$ & $1,00(0,75)$ & $1,75(0,63)$ & $1,25(1,00)$ & 0,110 \\
\hline Confronto com adversidade & $1,75(0,88)$ & $2,00(0,87)$ & $2,00(1,00)$ & $1,75(0,50)$ & 0,249 \\
\hline Concentração & $1,75(0,75)$ & $2,25(0,92)$ & $1,75(0,75)$ & $1,50(0,75)$ & 0,129 \\
\hline Formulação de objetivos & $2,25(0,38)$ & $2,25(0,50)$ & $2,37(0,62)$ & $2,25(1,00)$ & 0,732 \\
\hline Confiança e motivação para a realização & $2,25(0,75)$ & $2,50(0,63)$ & $2,75(1,00)$ & $2,12(0,75)$ & 0,115 \\
\hline Treinabilidade & $1,50(1,50)$ & $1,50(0,87)$ & $2,00(1,00)$ & $1,50(1,00)$ & 0,522 \\
\hline Índice de confronto no esporte & $7,36(1,97)$ & $8,00(2,36)$ & $8,14(3,50)$ & $7,14(1,29)$ & 0,137 \\
\hline
\end{tabular}

272 • Rev Bras Educ Fís Esporte, (São Paulo) 2013 Abr-Jun; 27(2):269-76 
É possível observar na TABELA 5 que atletas que treinam entre seis a 14 anos apresentam maior rendimento máximo sob pressão do que aqueles entre um a cinco anos de treinamento $(\mathrm{p}=0,002)$. Em relação ao confronto com adversidade, atletas que treinam entre seis e 14 anos apresentam maiores valores do que aqueles que treinam entre um e cinco anos $(p=0,004)$ e menores valores do que quem treina há mais de 15 anos $(\mathrm{p}=0,019)$. O grupo de atletas que treina há mais de 15 anos obteve menores valores de treinabilidade quando comparados com atletas que treinam entre um a cinco anos ( $\mathrm{p}$ $=0,005)$ e seis a 14 anos $(p=0,024)$. O grupo que treina entre seis a 14 anos apresentou valores mais baixos de índice de confronto no esporte em relação aos que treinam entre um e cinco anos $(\mathrm{p}=0,008)$ e acima de 15 anos $(0,013)$.

Notou-se na TABELA 6 que todas as correlações significativas entre "burnout" e estratégias de "coping" foram negativas, indicando que, quanto maior o "burnout" do indivíduo, menor é a frequência de utilização de estratégias de enfrentamento.

TABELA 5 - Comparação das estratégias de "coping” em relação ao tempo de prática de atletas brasileiros de vôlei de praia.

\begin{tabular}{|c|c|c|c|c|}
\hline \multirow{3}{*}{ "Coping" } & \multicolumn{3}{|c|}{ Tempo de prática } & \multirow{3}{*}{$\mathrm{p}$} \\
\hline & $1-5$ anos & 6-14 anos & $>15$ anos & \\
\hline & Md (IIQ) & Md (IIQ) & Md (IIQ) & \\
\hline Rendimento máximo sob pressão & $1,75(1,00)^{\mathrm{a}}$ & $2,00(0,75)^{\mathrm{a}}$ & $2,00(0,75)$ & ${ }^{\text {a }} 0,002^{*}$ \\
\hline Ausência de preocupaçôes & $1,50(1,00)$ & $1,00(0,50)$ & $1,50(1,12)$ & 0,609 \\
\hline Confronto com adversidade & $1,75(0,75)^{\mathrm{a}}$ & $2,00(0,50)^{\mathrm{a}, \mathrm{b}}$ & $2,00(0,87)^{\mathrm{b}}$ & $\begin{array}{l}\text { a } 0,004^{*} \\
\text { b } 0,019^{*}\end{array}$ \\
\hline Concentração & $1,75(0,75)^{\mathrm{a}}$ & $1,75(0,75)^{\mathrm{a}, \mathrm{b}}$ & $1,75(0,88)^{\mathrm{b}}$ & $\begin{array}{l}\text { a } 0,002^{*} \\
\text { b } 0,005^{*}\end{array}$ \\
\hline Formulação de objetivos & $2,25(1,00)$ & $2,25(0,50)$ & $2,25(0,63)$ & 0,323 \\
\hline Confiança e motivação para a realização & $2,25(1,00)$ & $2,25(0,75)$ & $2,50(0,75)$ & 0,092 \\
\hline Treinabilidade & $2,00(1,25)^{\mathrm{a}}$ & $1,50(0,25)^{\mathrm{b}}$ & $1,50(1,12)^{\mathrm{a}, \mathrm{b}}$ & $\begin{array}{l}\text { a } 0,005^{*} \\
\text { b } 0,024^{*}\end{array}$ \\
\hline Índice de confronto no esporte & $7,43(1,86)^{\mathrm{a}}$ & $7,29(1,57)^{\mathrm{a}, \mathrm{b}}$ & $7,71(1,07)^{\mathrm{b}}$ & $\begin{array}{l}\text { a } 0,008^{*} \\
\text { b } 0,013^{*}\end{array}$ \\
\hline
\end{tabular}

TABELA 6 - Correlação entre as estratégias de “coping” e dimensões de "burnout” em atletas brasileiros de vôlei de praia.

\begin{tabular}{|c|c|c|c|c|}
\hline \multirow[b]{2}{*}{ "Coping" } & \multicolumn{4}{|c|}{ "Burnout" } \\
\hline & $\begin{array}{c}\text { "Burnout" } \\
\text { total }\end{array}$ & $\begin{array}{c}\text { Exaustáo física } \\
\text { e emocional }\end{array}$ & $\begin{array}{c}\text { Reduzido senso } \\
\text { de realizaçáo }\end{array}$ & $\begin{array}{c}\text { Desvalorização } \\
\text { esportiva }\end{array}$ \\
\hline Rendimento máximo sob pressão & $-0,25^{* *}$ & $-0,07$ & $-0,30^{* *}$ & $-0,37^{* *}$ \\
\hline Ausência de preocupaçôes & 0,02 & 0,04 & $-0,04$ & 0,05 \\
\hline Confronto com adversidade & $-0,37^{* *}$ & $-0,28^{* *}$ & $-0,24^{*}$ & $-0,41^{* *}$ \\
\hline Concentração & $-0,27^{* *}$ & $-0,21^{*}$ & $-0,14$ & $-0,34^{* *}$ \\
\hline Formulação de objetivos & $-0,14$ & $-0,12$ & $-0,05$ & $-0,19$ \\
\hline Confiança e motivação para a realização & $-0,12$ & $-0,08$ & $-0,05$ & $-0,20$ \\
\hline Treinabilidade & $-0,05$ & $-0,06$ & $-0,01$ & $-0,03$ \\
\hline Índice de confronto no esporte & $-0,028^{* *}$ & $-0,18$ & $-0,21^{*}$ & $-0,35^{* *}$ \\
\hline
\end{tabular}




\section{Discussão}

Os principais achados indicaram que atletas com maior síndrome de "burnout" são aqueles que utilizam com menor frequência estratégias de "coping" e estão entre os piores ranqueados. Além disso, atletas do gênero masculino e com maior tempo de prática utilizam mais estratégias de "coping". Já com relação à categoria, atletas da categoria "open" utilizam mais estratégias de rendimento máximo sob pressão, enquanto os da categoria sub-21 são menos preocupados e mais dispostos a treinar.

A relação inversa entre síndrome de "burnout" e estratégias de "coping" (TABELA 6) recebe suporte na ideia de que pessoas que percebem as situaçōes estressantes de forma mais positiva e apresentam recursos de enfrentamento cognitivos e comportamentais lidam melhor com os fatores que poderiam gerar um estresse crônico e, consequentemente, tem uma menor propensão em desenvolver o "burnout"22-23.

Por outro lado, estratégias de "coping" como ausência de preocupações, formulação de objetivos, confiança e motivação e treinabilidade não apresentaram correlação com nenhuma das dimensões do "burnout", o que não indica que essas estratégias não sejam utilizadas de forma eficaz pelos atletas em algum momento do ciclo de treinamento, uma vez que as estratégias de enfrentamento podem mudar ao longo da temporada ${ }^{24}$.

O confronto com a adversidade parece ser a estratégia de "coping" mais importante utilizada pelos atletas para enfrentar as situações estressantes, tendo em vista que se correlacionou com todas as dimensōes do "burnout" e também com desvalorização esportiva (TABELA 6). Este achado vai ao encontro do estudo qualitativo de DiAs et al. ${ }^{16} \mathrm{com}$ atletas de elite portugueses, o qual apontou que as principais estratégias de "coping" utilizadas estavam relacionadas ao "coping" ativo, sobretudo, com foco no esforço e trabalho, tentativas de resolução de problemas e não desistir de lutar.

É importante ressaltar que as relações entre as dimensôes do "burnout" e as estratégias de "coping" devem ser analisadas com cautela, uma vez que os valores das correlações, ainda que significativos, são considerados baixos e moderados ${ }^{25}$.

A comparação entre os gêneros indicou que os atletas do gênero masculino utilizaram com maior frequência todas as estratégias de "coping" quando comparados com o gênero feminino (TABELA 2), resultado semelhante ao encontrado com atletas e estudantes americanos, no qual os homens apresentaram maiores estratégias de enfrentamento em relação às mulheres ${ }^{10}$.

Por outro lado, com relação ao "burnout", embora a maioria dos estudos tenha apontado que as mulheres apresentam mais sintomas de estresse ${ }^{26-27}$, no presente estudo, não foram encontradas diferenças significativas entre gênero nas dimensões do "burnout" (TABELA 1). Para MARQues et al..$^{23}$, as diferenças nas percepções das situações estressantes podem estar vinculadas muito mais a diferenças no perfil psicológico das pessoas, como características de personalidade ${ }^{28}$ e de motivação ${ }^{29}$, do que simplesmente ao gênero. Talvez seja esta a explicação de também não terem sido encontradas diferenças significativas entre as dimensões do "burnout" em função da categoria e do tempo de prática (TABELA 1).

Notou-se que os atletas acima da $9^{a}$ colocação foram os que apresentaram maiores frequências de comportamentos vinculados às dimensões: reduzido senso de realização esportiva, desvalorização da modalidade esportiva e valor total de "burnout". PIRES et al. 5 esclarecem que o "burnout" em atletas é decorrente da incompatibilidade dos planos e metas iniciais dos mesmos na modalidade esportiva com as demandas crônicas de cunho sócio-psico-físico do próprio esporte. Tendo em vista que os atletas piores colocados podem ser aqueles que enfrentam constantemente as situações mais apontadas como estressantes como cometer mais erros, perceber-se com grandes limitações de capacidades e habilidades e receber cobranças frequentes de treinadores e patrocinadores pelos maus resultados ${ }^{15-16}$, pode ser que se sintam mais propensos a ter suas expectativas frustradas ${ }^{8,30} \mathrm{e}$, consequentemente, podem apresentar maiores índices de "burnout".

Os atletas acima do $9^{\circ}$ lugar também foram aqueles que utilizaram com menos frequência a estratégia de rendimento máximo sob pressão (TABELA 4) e talvez este seja um dos fatores relacionados ao baixo resultado, uma vez que a competição é apontada como a principal situação geradora de estresse ${ }^{13}$. Além disso, atletas da categoria sub-21 apresentaram maior frequência de utilização de estratégias de coping vinculadas à ausência de preocupação e treinabilidade, um dado esperado, tendo em vista que estes atletas treinam para futuramente competir na categoria "open", considerada a principal do circuito brasileiro de vôlei de praia.

Por outro lado, atletas da categoria "open" (TABELA 3) e que apresentam maior tempo de prática 
(TABELA 5) foram aqueles que obtiveram maior frequência de utilização de rendimento máximo sob pressão e de outras estratégias de "coping", o que encontra suporte na afirmação de que habilidades psicológicas podem ser desenvolvidas com a prática esportiva e, portanto, necessitam ser trabalhadas ao longo da formação do atleta ${ }^{31-32}$.

As limitaçōes do presente estudo referem-se a de um estudo transversal, uma vez que, pelo fato de ter sido desenvolvido com atletas que estavam participando de uma etapa do circuito brasileiro de vôlei de praia, não foi possível identificar se os sintomas de "burnout" e as estratégias de "coping" se apresentam de forma diferenciada ao longo do ano, e esta poderia ser uma sugestão para estudos futuros. No entanto, por ser uma pesquisa original, a exploração dos dados descritivos possibilitou entendimento desta temática envolvendo atletas de vôlei de praia. Outra limitação, é que as informaçōes foram obtidas através de questionários que, embora sejam validados e amplamente utilizados com atletas, não avaliam as possíveis interaçōes entre "burnout" e "coping", limitando-se a uma inferência estatística. Assim, novos estudos poderiam complementar estas informações com abordagens qualitativas.

Concluiu-se que quanto maior a utilização de estratégias de "coping", menor são os sintomas de "burnout" apresentados e, atletas do gênero masculino, melhores colocados no "ranking", da categoria "open" e com maior tempo de treinamento se utilizam com mais frequência de diferentes estratégias de "coping" para combater a síndrome de "burnout".

\begin{abstract}
Analysis of burnout syndrome and coping strategies in Brazilian athletes beach volleyball

This study aimed to analyze burnout syndrome and coping strategies of beach volleyball players. Ninetythree athletes participated, being one group of under-21 age and another group of adult category. The both group are composed by players of the Brazilian beach volleyball championship. The instruments used were a form with data identification, Burnout Questionnaire for Athletes and Athletic Inventory of Coping Strategies. For data analysis, the Kolmogorov-Smirnov test, Cronbach's alpha coefficient, 'U' Mann-Whitney test, Kruskal-Wallis test and Spearman correlation coefficient, were used, considering $p$ $<0.05$. There was a significant and negative correlation between the dimensions of burnout and coping strategies. For the dimensions of burnout, just ranking obtained significant difference, while for coping strategies, gender, category, ranking and practice time showed significant differences. It was concluded that the higher burnout, fewer beach volleyball athletes use coping strategies.
\end{abstract}

KEY WORDS: Sports psychology; Emotions; Burnout professional; Coping strategies.

\title{
Referências
}

1. Trigo TR, Teng CT, Hallak JEC. Síndrome de burnout ou estafa profissional e os transtornos psiquiátricos. Rev Psiquiatr Clin. 2007;34:223-33.

2. Carlotto MS, Câmara SG. Análise da produção científica sobre a síndrome de burnout no Brasil. Psico. 2008;39:152-8.

3. Gould D, Whitley MA. Sources and consequences of athletic burnout among college athletes. J Intercollegiate Sports. 2009;2:16-30.

4. Kania ML, Meyer BB, Ebersole KT. Personal and environmental characteristics predicting burnout among certified athletic trainers at National Collegiate Athletic Association Institutions. J Athl Train. 2009;44:58-66.

5. Pires DA, Brandão MRF, Silva CB. Validação do questionário de burnout para atletas. Rev Educ Fis. 2006;17:27-36.

6. Chiminazzo JGC, Montagner PC. Síndrome de burnout e esporte: a visão dos técnicos de tênis de campo. Rev Educ Fis. 2009;20:217-23.

7. Gustafsson H, Kenttä G, Hassmén P, Lundqvist C. Prevalence of burnout in competitive adolescent athletes. Sport Psychol. 2007;21:21-37. 
8. Gustafsson H, Hassmén P, Kenttä G, Johansson M. A qualitative analysis of burnout in elite Swedish athletes. Psychol Sport Exerc. 2008;9:800-16.

9. Gomes R. Adaptação humana em contextos desportivos: contributos da teoria para a avaliação psicológica. Aval Psicol. 2011;10:13-24.

10. Anshel MH, Sutarso T, Jubenville C. Racial and gender differences on sources off acute stress and coping style among competitive athletes. J Soc Psychol. 2009;149:159-77.

11. Nicholls A, Polman R, Morley D, Taylor NJ. Coping and coping effectiveness in relation to a competitive sport event: pubertal status, chronological age, and gender among adolescent athletes. J Sport Exerc Psychol. 2009;31:299-317.

12. Rosado A, Mesquita I, Correia A, Colaço C. Relação entre esgotamento e satisfação em jovens praticantes desportivos. Rev Port Cienc Desporto. 2009;9:56-67.

13. De Rose Junior D. A competição como fonte de estresse no esporte. Rev Bras Cienc Mov. 2002;10:19-26.

14. Marques ACP, Rosado AFP. Situações estressantes nos atletas de basquetebol de alta competição. Rev Bras Educ Fís Esporte. 2005;19:71-87.

15. De Rose Junior D, Sato CT, Selingardi D, Bettencourt EL, Barros JCTS, Ferreira MCM. Situações de jogo como fonte de stress em modalidades esportivas coletivas. Rev Bras Educ Fís Esporte. 2004;18:385-95.

16. Dias C, Cruz JF, Fonseca AM. Emoçōes, stress, ansiedade e coping: estudo qualitativo com atletas de elite. Rev Port Cienc Desporto. 2009;9:9-23.

17. Stefanello J. Situaçôes de estresse no vôlei de praia de alto rendimento: um estudo de caso com uma dupla olímpica. Rev Port Cienc Desporto. 2007;7:232-44.

18. Thomas JR, Nelson JK, Silverman SJ. Métodos de pesquisa em atividade física. 5a ed. Artmed: Porto Alegre; 2007.

19. Raedeke T, Smith A. Development and preliminary validation of an athlete burnout measure. J Sport Exerc Psychol. 2001;23:281-306.

20. Smith RE, Schutz RW, Smoll FL, Ptacek JT. Development and validation of a multidimensional measure of sportspecific psychological skills: the Athletic Coping Skills Inventory-28. J Sport Exerc Psychol. 1995;17:379-98.

21. Serpa S, Palmeira AL. ACSI 28: estudo das experiências desportivas. Lisboa: Laboratório de Psicologia do desporto/ Faculdade de Motricidade Humana/Universidade Técnica de Lisboa; 1997.

22. Lazarus RS, Folkman S. Estresse, appraisal, and coping. New York: Springer; 1984.

23. Marques RS, Cipriani M, Melo GF, Giavoni A. Os níveis de estresse pré-competitivo de atletas classificados segundo a tipologia dos esquemas de gênero. Motriz. 2010;16:59-68.

24. Tamminen KA, Holt NL. Female adolescent athletes' coping: a season-long investigation. J Sports Sci. 2010;28:101-14.

25. Dancey CP, Reidy J. Estatística sem matemática para a psicologia. 3a ed. Artmed: Porto Alegre; 2008.

26. Costa M, Accioly Júnior H, Oliveira J, Maia E. Estresse: diagnóstico dos policiais militares em uma cidade brasileira. Rev Panam Salud Publica. 2007;21:217-22.

27. Gomes AR, Montenegro N, Peixoto AMBC, Peixoto ARBC. Stress ocupacional no ensino: um estudo com professores dos $3^{\circ}$ ciclo e ensino secundário. Psico Soc. 2010;22:587-97.

28. Hill AP, Hall HK, Appleton PR, Murray JJ. Perfectionism and burnout in canoe polo and kayak slalom athletes: the mediating influence of validation and growth-seeking. Sport Psychol. 2010;24:16-34.

29. Lonsdale C, Hodge K, Rose E. Athlete burnout in elite sport: a self-determination perspective. J Sports Sci. 2009;27:785-95.

30. O’Neil JW, Steyn BJM. Strategies used by south African non-elite athletes to cope with the environmental stressors associated with endurance events. S Afr J Res Sport Phys Educ Recreat. 2007;29:99-107.

31. Weinberg RS, Gould D. Fundamentos da psicologia do esporte e do exercício. 4a ed. Artmed: Porto Alegre; 2008.

32. Stefanello J. Competências psicológicas no vôlei de praia de alto rendimento: síntese e recomendaçôes para o treinamento. Motriz. 2009;15:996-1008.

\begin{tabular}{r|l} 
ENDEREÇo & \\
Patrícia Aparecida Gaion Rigoni & \\
Departamento de Educação Física & Recebido para publicação: 03/01/2012 \\
Universidade Estadual de Maringá & 1a. Revisão: 19/11/2012 \\
Av. Colombo, 5790 & 2a. Revisão: 04/02/2013 \\
87020-900 - Maringá - PR - BRASIL & Aceito: 08/02/2013 \\
e-mail: pagrigoni@uem.br &
\end{tabular}

276 • Rev Bras Educ Fís Esporte, (São Paulo) 2013 Abr-Jun; 27(2):269-76 